\title{
ANALYSIS OF LARGE-SCALE ANISOTROPY OF ULTRA-HIGH ENERGY COSMIC RAYS IN HiRes DATA
}

\author{
R. U. Abbasi ${ }^{1}$, T. Abu-Zayyad ${ }^{1}$, M. Allen ${ }^{1}$, J. F. Amann ${ }^{2}$, G. Archbold ${ }^{1}$, K. Belov ${ }^{1}$, J. W. Belz ${ }^{1}$, D. R. Bergman , $^{1,3}$,

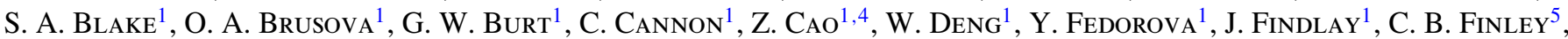 \\ R. C. Gray ${ }^{1}$, W. F. Hanlon ${ }^{1}$, C. M. Hoffman ${ }^{2}$, M. H. Holzscheiter ${ }^{2}$, G. Hughes ${ }^{3}$, P. HüntemeyeR ${ }^{1}$, D. Ivanov ${ }^{3}$, B. F Jones ${ }^{1}$, \\ C. C. H. Jui ${ }^{1}$, K. Kim ${ }^{1}$, M. A. Kirn ${ }^{6}$, H. Koers ${ }^{7}$, E. C. LoH $^{1}$, M. M. Maestas ${ }^{1}$, N. Manago ${ }^{8}$, L. J. MareK ${ }^{2}$, K. Martens ${ }^{1}$, \\ J. A. J. Matthews ${ }^{9}$, J. N. Matthews ${ }^{1}$, S. A. Moore ${ }^{1}$, A. O’Neill ${ }^{5}$, C. A. Painter ${ }^{2}$, L. Perera ${ }^{3}$, K. Reil ${ }^{1}$, R. Riehle ${ }^{1}$, \\ M. D. Roberts ${ }^{9}$, D. Rodriguez ${ }^{1}$, M. SASAKI ${ }^{8}$, S. R. SchnetZer ${ }^{3}$, L. M. Scott ${ }^{3}$, G. Sinnis ${ }^{2}$, J. D. Smith ${ }^{1}$, P. Sokolsky ${ }^{1}$, \\ C. Song $^{5}$, R. W. Springer ${ }^{1}$, B. T. Stokes ${ }^{1,3}$, S. R. Stratton ${ }^{3}$, J. R. Thomas ${ }^{1}$, S. B. Thomas ${ }^{1}$, G. B. Thomson $^{1,3}$, P. Tinyakov $^{7}$, \\ D. TuPA ${ }^{2}$, L. R. WIENCKE ${ }^{1}$, A. ZECH ${ }^{3}$, AND X. ZHANG ${ }^{5}$ \\ (The High Resolution Fly's Eye Collaboration) \\ ${ }^{1}$ Department of Physics and High Energy Astrophysics Institute, University of Utah, Salt Lake City, UT 84112, USA \\ ${ }^{2}$ Los Alamos National Laboratory, Los Alamos, NM 87545, USA \\ ${ }^{3}$ Department of Physics and Astronomy, Rutgers-The State University of New Jersey, Piscataway, NJ 08854, USA \\ ${ }^{4}$ Institute of High Energy Physics, Beijing, China \\ ${ }^{5}$ Department of Physics and Nevis Laboratories, Columbia University, New York, NY 10027, USA \\ ${ }^{6}$ Department of Physics, Montana State University, Bozeman, MT, USA \\ ${ }^{7}$ Université Libre de Bruxelles (ULB), CP225, Bld. du Triomphe, B-1050 Brussels, Belgium \\ ${ }^{8}$ Institute for Cosmic Ray Research, University of Tokyo, Kashiwa City, Chiba 277-8582, Japan \\ ${ }^{9}$ Department of Physics and Astronomy, University of New Mexico, Albuquerque, NM 87131, USA \\ Received 2010 February 5; accepted 2010 March 5; published 2010 March 25
}

\begin{abstract}
Stereo data collected by the HiRes experiment over a six-year period are examined for large-scale anisotropy related to the inhomogeneous distribution of matter in the nearby universe. We consider the generic case of small cosmic-ray deflections and a large number of sources tracing the matter distribution. In this matter tracer model the expected cosmic-ray flux depends essentially on a single free parameter, the typical deflection angle $\theta_{s}$. We find that the HiRes data with threshold energies of $40 \mathrm{EeV}$ and $57 \mathrm{EeV}$ are incompatible with the matter tracer model at a $95 \%$ confidence level unless $\theta_{s}>10^{\circ}$ and are compatible with an isotropic flux. The data set above $10 \mathrm{EeV}$ is compatible with both the matter tracer model and an isotropic flux.
\end{abstract}

Key words: cosmic rays - large-scale structure of universe - magnetic fields

\section{INTRODUCTION}

The observation of the cutoff in the spectrum of Ultra-High Energy Cosmic Rays (UHECRs; Abbasi et al. 2008a; Abraham et al. 2008a) as predicted by Greisen (1966) and Zatsepin \& Kuzmin (1966) provides compelling evidence for the shortening of the UHECR propagation length at high energies. The highest energy events then must have come from relatively close sources (within $250 \mathrm{Mpc}$ ). At these length scales, the matter in the universe is distributed inhomogeneously, being organized into clusters and superclusters. One should, therefore, expect the flux of highest energy cosmic rays to be anisotropic.

In astrophysical scenarios, it is natural to assume that the number of sources within $250 \mathrm{Mpc}$ is large and that these sources trace the distribution of matter. Under these assumptions, the anisotropy at Earth depends only on the nature and size of UHECR deflections. Measurement of the anisotropy, therefore, gives direct experimental access to parameters that determine the deflections, notably to the UHECR charge composition and cosmic magnetic fields.

Several investigations into anisotropy in arrival directions of UHECRs previously have been undertaken. At small angular scales, correlations with different classes of putative sources were claimed (e.g., Gorbunov et al. 2004; Abbasi et al. 2006; Abraham et al. 2007, 2008b). At larger angular scales and energies below $10 \mathrm{EeV}$, possible anisotropy toward the Galactic center was reported in Hayashida et al. (1999) and Bellido et al. (2001), but not supported by more recent studies (Santos 2007).
At higher energies, Stanev et al. (1995) found evidence against an isotropic flux above $40 \mathrm{EeV}$ through correlations with the supergalactic plane, but this was not confirmed by other authors (Hayashida et al. 1996; Kewley et al. 1996; Bird et al. 1999). Finally, using the Pierre Auger Observatory (PAO) data, Kashti \& Waxman (2008) have found correlations between UHECR arrival directions and the large-scale structure of the universe, which are incompatible with an isotropic flux (see, however, Koers \& Tinyakov 2009a).

In this Letter, we analyze the data accumulated by the HiRes experiment for anisotropy associated with the large-scale structure of the universe. The HiRes experiment has been described previously (Abu-Zayyad et al. 1999; Boyer et al. 2002; Hanlon 2008). It studied UHECRs from $10^{17.2} \mathrm{eV}$ to $10^{20.2} \mathrm{eV}$ using the fluorescence technique. HiRes operated two fluorescence detectors located atop desert mountains in west-central Utah. The data set used in this study consists of events observed by both detectors, analyzed jointly in what is commonly called "stereo mode." In this mode, the angular resolution in cosmic rays' pointing directions is about 0.8 , and the energy resolution is about $10 \%$. The HiRes experiment operated in the stereo mode between 1999 December and 2006 April. At the highest energies, HiRes has the largest data set in the Northern hemisphere. A large number of events, good angular resolution (better than the bending angles expected from Galactic and extragalactic magnetic fields), and the wide energy range covered make the HiRes data particularly suitable for anisotropy studies. The exact data set 

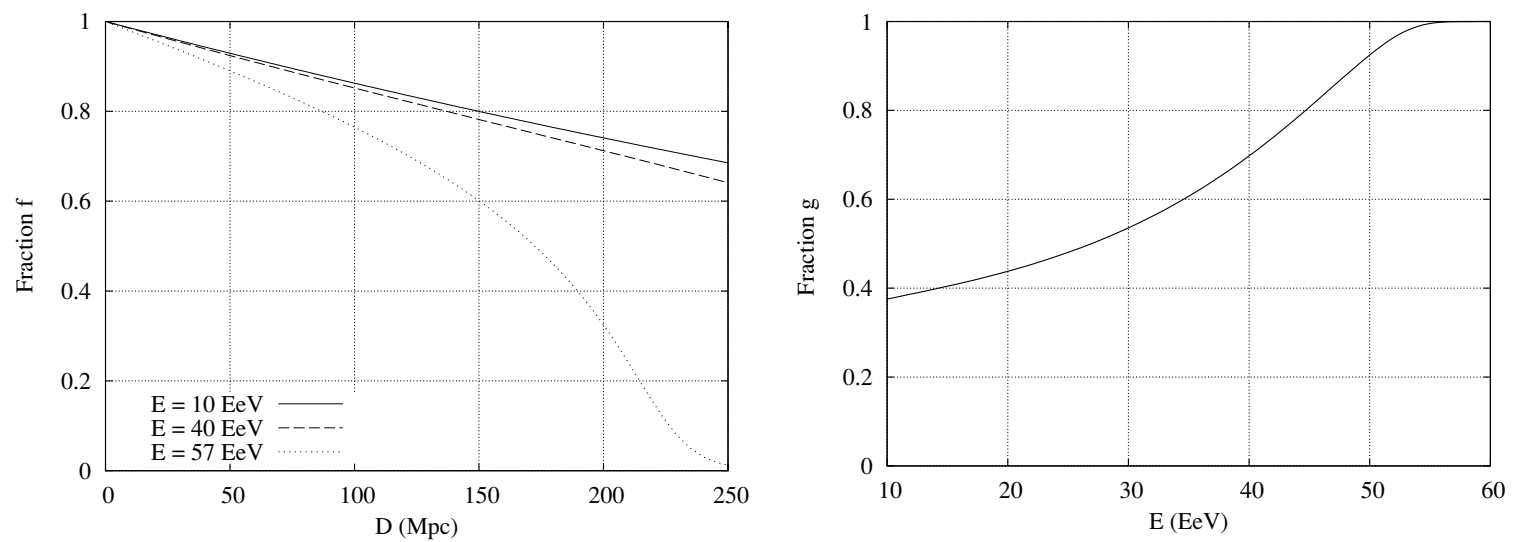

Figure 1. Left panel: fraction $f$ of integral CR flux that survives after interactions with the CMB and cosmological redshift as a function of distance $D$ for threshold energies $10 \mathrm{EeV}$ (solid line), $40 \mathrm{EeV}$ (dashed), and $57 \mathrm{EeV}$ (dotted). Right panel: fraction $g$ of total flux that is produced by sources within $250 \mathrm{Mpc}$, as a function of energy $E$.

used in this study was described previously in Abbasi et al. (2008b).

We consider here a generic model that assumes many sources within $250 \mathrm{Mpc}$ tracing the distribution of matter, which we refer to as the "matter tracer" model. We also assume that deflections of UHECR do not exceed the angular size of the nearby structures that is $10^{\circ}-20^{\circ}$. In this regime, both regular and random deflections in magnetic fields can be modeled with a one-parameter distribution, for which we take a Gaussian distribution centered at zero with width $\theta_{\mathrm{s}}$. This width is treated as a free parameter, whose value we aim to constrain from the data. Constraints on $\theta_{\mathrm{s}}$ may then be used to obtain information on the strength of Galactic and extragalactic magnetic fields. In keeping with our assumption of small deflections, we assume a proton composition in this study, which is consistent with the $X_{\max }$ analysis based on the same data set (for confirmation see Abbasi et al. 2009).

The HiRes data are compared to model predictions using the "flux sampling" test put forward by Koers \& Tinyakov (2009a). This test has good discrimination power at small statistics and is insensitive to the details of deflections. The comparison is performed at three different threshold energies that have been used in previous studies: $10 \mathrm{EeV}, 40 \mathrm{EeV}$, and $57 \mathrm{EeV}$ (Hayashida et al. 1996; Abbasi et al. 2006; Abraham et al. 2007). An a priori significance of $5 \%$, corresponding to a confidence level (CL) of $95 \%$, is chosen for this work.

This Letter is organized as follows. In Section 2, we discuss the modeling of UHECR arrival directions. Section 3 concerns the HiRes data used in the analysis, while Section 4 describes the flux sampling method. We present our results in Section 5 and conclude in Section 6.

\section{MODELING OF UHECR ARRIVAL DIRECTIONS}

Galaxy catalog. The distribution of matter in the local universe is modeled with the 2 Micron All-Sky Redshift Survey (2MRS; J. Huchra et al. 2010, in preparation) galaxy catalog, using galaxies as samplers of the underlying matter density field. ${ }^{10}$ The 2MRS is a flux-limited sample of galaxies, that is, the sample containing all galaxies with observed magnitude in the $K_{s}$ band $m \leqslant 11.25$. It contains spectroscopically measured redshifts for all but a few galaxies. A number of cuts have been applied to the galaxy sample. First, the Galactic plane, where

\footnotetext{
10 This sample was kindly provided by John Huchra.
}

the sample is incomplete, has been excluded from the sample by removing all galaxies with $|b|<10^{\circ}$. Second, objects with $D<5 \mathrm{Mpc}$ are removed because such objects should be treated on an individual basis. ${ }^{11}$ Finally, the catalog is cut at $250 \mathrm{Mpc}$ because the sample becomes too sparse. The resulting sample provides an accurate statistical description at smearing angles $\theta_{s}>2^{\circ}$. The flux from sources beyond $250 \mathrm{Mpc}$ is taken to be isotropic. A total of 15508 galaxies remain in the HiRes field of view after the cuts. To compensate for observational selection effects in the (flux-limited) 2MRS catalog, weights $w_{i}^{\text {cat }}$ are assigned to the galaxies with the sliding-box method as described in Koers \& Tinyakov (2009b).

Energy loss. UHECR fluxes are affected by energy loss due to redshift and interactions with the cosmic microwave background (CMB). To account for the resulting flux suppression, the integral flux, $\varphi_{i}$, from a single source is expressed as follows:

$$
\varphi_{i}\left(E, D_{i}\right)=\frac{J(E) f\left(E, D_{i}\right)}{4 \pi D_{i}^{2}},
$$

where $E$ is the threshold energy, $D_{i}$ is the source distance, $J$ stands for the integral injection spectrum at the source, and $f$ represents the flux fraction that remains after interactions and redshift. We take an injection spectrum $J(E) \propto E^{-1.2}$ extending to very high energies. The function $f$ is determined using a numerical propagation code based on the continuous loss approximation that is described in Koers \& Tinyakov (2008, 2009a). Energy loss due to interactions with the extragalactic background light is neglected. In Figure 1, left panel, the fraction $f$ is shown as a function of distance for the different energies considered in this work.

The strength of the isotropic flux component that is added to account for sources beyond $250 \mathrm{Mpc}$ also depends on UHECR energy loss. Using the computer code described in the previous paragraph, we estimate the fraction $g$ of total flux contributed by sources within $250 \mathrm{Mpc}$ to be $0.4,0.7$, and 1.0 for threshold energies $E=10 \mathrm{EeV}, 40 \mathrm{EeV}$, and $57 \mathrm{EeV}$, respectively (see Figure 1, right panel).

Deflections. UHECR protons (as well as nuclei) are deflected by Galactic and intergalactic magnetic fields. These deflections are taken into account by an angular smearing procedure, which replaces the point-source flux, $\varphi$, by a flux density distribution,

$$
\varphi_{i} \rightarrow \varphi_{i} w^{s}\left(\theta_{i}\right)
$$

11 This corresponds to the ad hoc assumption that there are no UHECR sources within $5 \mathrm{Mpc}$. Different analyses are more appropriate to test this possibility. 
where $w^{s}\left(\theta_{i}\right)$ represents the probability density that an UHECR is deflected by $\theta_{i}$, the angle between galaxy $i$ and the line of sight. This procedure also accounts for the detector's angular resolution and prevents unphysical fluctuations due to the use of a catalog of point sources. In the absence of detailed knowledge about the structure of Galactic and extragalactic magnetic fields, we adopt a simple Gaussian probability density distribution with characteristic angle, $\theta_{\mathrm{s}}$. This angle is treated as a free model parameter. The Gaussian distribution is a fair approximation when the deflections are small. For large deflections, details on the structure of the Galactic and extragalactic magnetic fields become important. Accounting for these details goes beyond the scope of the present study.

Exposure. The HiRes exposure is modeled using our Monte Carlo simulation of the experiment (Abbasi et al. 2007; Bergman 2007). The aim of this simulation was to create a set of Monte Carlo events that would be in all essences identical to the actual data. In making the simulation, we put in the properties of cosmic-ray air showers as measured by previous experiments (Bird et al. 1993; Abu-Zayyad et al. 2000, 2001; Abbasi et al. 2005). We used cosmic-ray showers generated by the Corsika and QGSJet programs (Heck et al. 1998; Kalmykov et al. 1997) and simulated the generation of fluorescence light (see references in Abbasi et al. 2008a) and its propagation through the atmosphere (see references in Abbasi et al. 2008a). A complete simulation of the optics and electronics (trigger and front-end electronics) of our detectors was performed. The result was an excellent simulation of our experiment as evidenced by the very good agreement between data and simulated events in the distribution of all kinematic variables, e.g., zenith angle, impact parameter to detector, etc. By assigning Monte Carlo events times of occurrence taken from the actual on-time of the experiment we are able to calculate the exposure on the sky very accurately.

Model flux maps. The integral UHECR flux from a given direction is expressed as follows:

$$
\Phi=\sum_{i} \varphi_{i} w_{i}^{\mathrm{cat}} w^{\mathrm{s}}\left(\theta_{i}\right)+\Phi_{\mathrm{iso}},
$$

where $i$ enumerates galaxies in the 2MRS sample, $w_{i}^{\text {cat }}$ denotes the weight assigned to galaxy $i$ in the catalog, $w^{\mathrm{s}}\left(\theta_{i}\right)$ is the deflection probability distribution, and $\Phi_{\text {iso }}$ is the UHECR flux arising from sources beyond $250 \mathrm{Mpc}$.

The probability to observe a cosmic ray (CR) from a given direction is proportional to the product of flux and exposure. We denote this probability as

$$
\widetilde{\Phi}=\Phi \Xi
$$

where $\Xi$ stands for exposure. In Figure 2, the distribution of $\widetilde{\Phi}$ over the sky is shown for three different threshold energies. The contrast in the flux distributions becomes more pronounced with increasing energy. Also shown are the arrival directions of UHECRs in the HiRes data to which the model flux has to be compared.

\section{DATA}

The data set used in this study was described previously in Abbasi et al. (2008b), including selection criteria and a correction to the energy scale. Our sample of the $2 \mathrm{MRS}$ catalog does not cover the region near the Galactic plane with $|b|<10^{\circ}$. We therefore removed cosmic-ray events with $|b|<10^{\circ}$ from
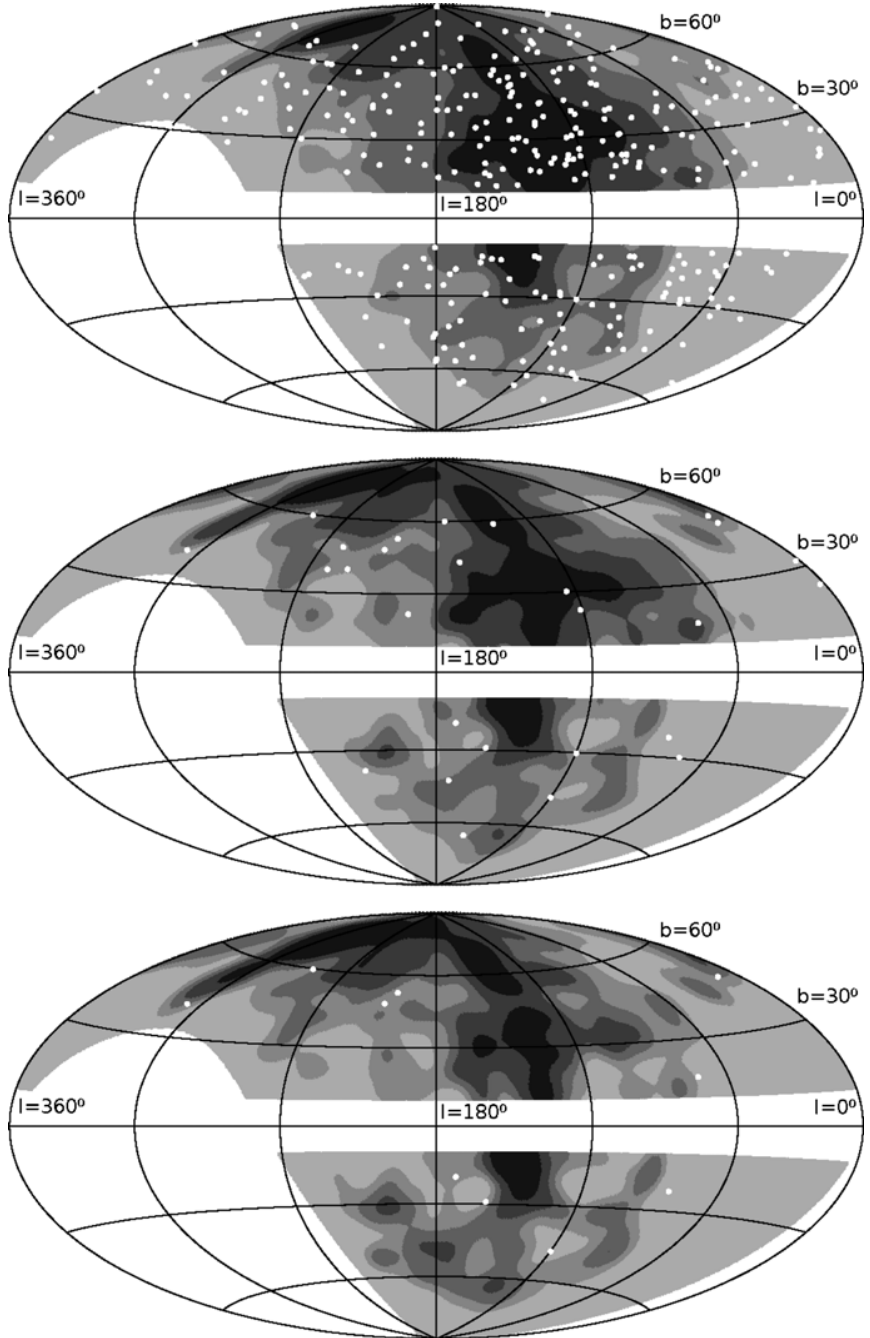

Figure 2. Hammer projection (galactic coordinates) of $\widetilde{\Phi}$ (flux times exposure) with threshold energies $10 \mathrm{EeV}$ (top panel), $40 \mathrm{EeV}$ (middle), and $57 \mathrm{EeV}$ (bottom). Darker gray indicates a higher value; the bands are chosen such that each band contains $1 / 5$ of the total flux (weighted with exposure). Excluded regions, namely, the galactic plane $\left(|b|<10^{\circ}\right)$ and the region outside the HiRes field of view, are shown in white. White dots indicate HiRes events. All maps are produced with $\theta_{\mathrm{s}}=6^{\circ}$.

the analysis. The resulting sample contains (1) 309 events with $E>10 \mathrm{EeV}$, (2) 27 events with $E>40 \mathrm{EeV}$, and (3) 10 events with $E>57 \mathrm{EeV}$. The arrival directions of these events are shown as white dots in Figure 2.

\section{STATISTICAL TEST}

The compatibility of a model flux map with the set of UHECR arrival directions is quantified by the flux sampling method introduced by Koers \& Tinyakov (2009a). The idea of the method is as follows. With any set of arrival directions one associates the set of flux values that are obtained by sampling a given flux map (such as the map shown in Figure 2), i.e., by extracting the flux values at the corresponding points on the sphere. The two-dimensional distribution of arrival directions thus translates into a one-dimensional distribution of flux values. If the reference model is true, this flux distribution will tend to high values since events fall preferentially into regions where the model flux (times exposure) is high. If, on the other hand, the reference model is not true, the flux 


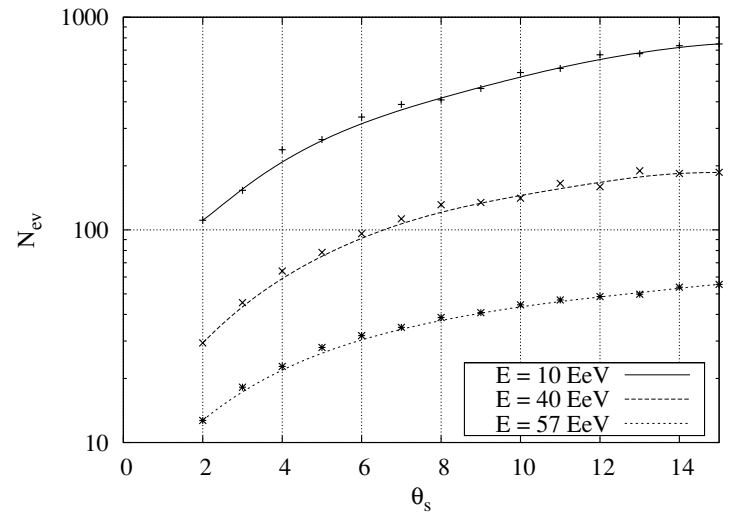

Figure 3. Number of events required for a $50 \%$ probability to rule out the matter tracer model at $95 \% \mathrm{CL}$ if the true flux is isotropic.

distribution is more uniform because the correlation between arrival directions and regions of high model flux is (partly) destroyed. By comparing the flux distribution to a model flux distribution, the compatibility between a set of arrival directions and model predictions can be quantified. This comparison is performed by the Kolmogorov-Smirnov test, which yields a test statistic $D$. The relevant statistical quantities, in particular powers and $p$-values, are computed from the distribution of this test statistic. Note that this test does not involve any additional parameters like bin size.

The ability of the test to discriminate between models may be quantified in terms of the statistical power, $P$, i.e., the probability of ruling out, at a given CL, the reference model when an alternative model is true. Within numerical uncertainties, the statistical power is equal to the fraction of event sets generated under the alternative model that lead to rejecting the reference model. Figure 3 shows the number of events required for a power $P=0.5$ (i.e., a $50 \%$ probability) to rule out the matter tracer model (at 95\% CL) when the true flux is isotropic. The number of events increases with increasing smearing angle and decreasing energy: the decreasing flux contrasts in the matter tracer model call for an increase in statistics to achieve the same discriminatory power. Observe that the event numbers indicated in Figure 3 are of the same order as the data analyzed in this work. We thus expect that there are sufficient data to obtain meaningful constraints at 95\% CL.

\section{RESULTS}

\subsection{Scan Over Smearing Angles}

The level of compatibility between data and model predictions is quantified by a $p$-value, which represents the model probability of obtaining a measurement that is at least as extreme as the actual measurement is. With our a priori choice of significance, a $p$-value smaller than 0.05 rules out the model. The probability of falsely excluding the model is then 5\%, translating into a CL of $95 \%$.

Figure 4 shows the $p$-values obtained by the flux sampling method for the HiRes data and predictions of the matter tracer model. The smearing angle, $\theta_{\mathrm{s}}$, is treated as a free parameter. That is, at each value of $\theta_{\mathrm{s}}$ and each threshold energy a flux map is generated and compared to the HiRes data as described above. The results can be summarized as follows.

1. For the threshold energies of $40 \mathrm{EeV}$ and $57 \mathrm{EeV}$, the tests show a disagreement between data and the matter tracer model for $\theta_{\mathrm{s}} \leqslant 10^{\circ}$. Within this parameter range, a source distribution tracing the distribution of matter is excluded at a $95 \%$ CL.

2. For the threshold energy of $10 \mathrm{EeV}$, the test shows an agreement between data and the matter tracer model.

The incompatibility between the data and matter tracer model is illustrated by the non-correlation between the observed arrival directions and regions of high model flux shown in the two lower panels of Figure 2.

We have also tested the data for compatibility with an isotropic model flux and found no disagreement, at 95\% CL, for any of the three tested threshold energies (the data with threshold energy $57 \mathrm{EeV}$ are marginally consistent with an isotropic flux).

\subsection{Case Study: $E=57 \mathrm{EeV}, \theta_{\mathrm{s}}=3.2$}

At energy threshold $E>57 \mathrm{EeV}$, a correlation between the arrival directions of UHECRs and the location of active galactic nuclei (AGNs) contained in the 12th edition of the Véron-Cetty \& Véron catalog (Véron-Cetty \& Véron 2006) was reported by the PAO (Abraham et al. 2007, 2008b). This correlation was found to be maximal for $\psi=3.2$, where $\psi$ denotes the maximum angular distance between UHECRs and AGNs. In the Northern hemisphere, correlation with AGN was not confirmed by the HiRes experiment (Abbasi et al. 2008b).
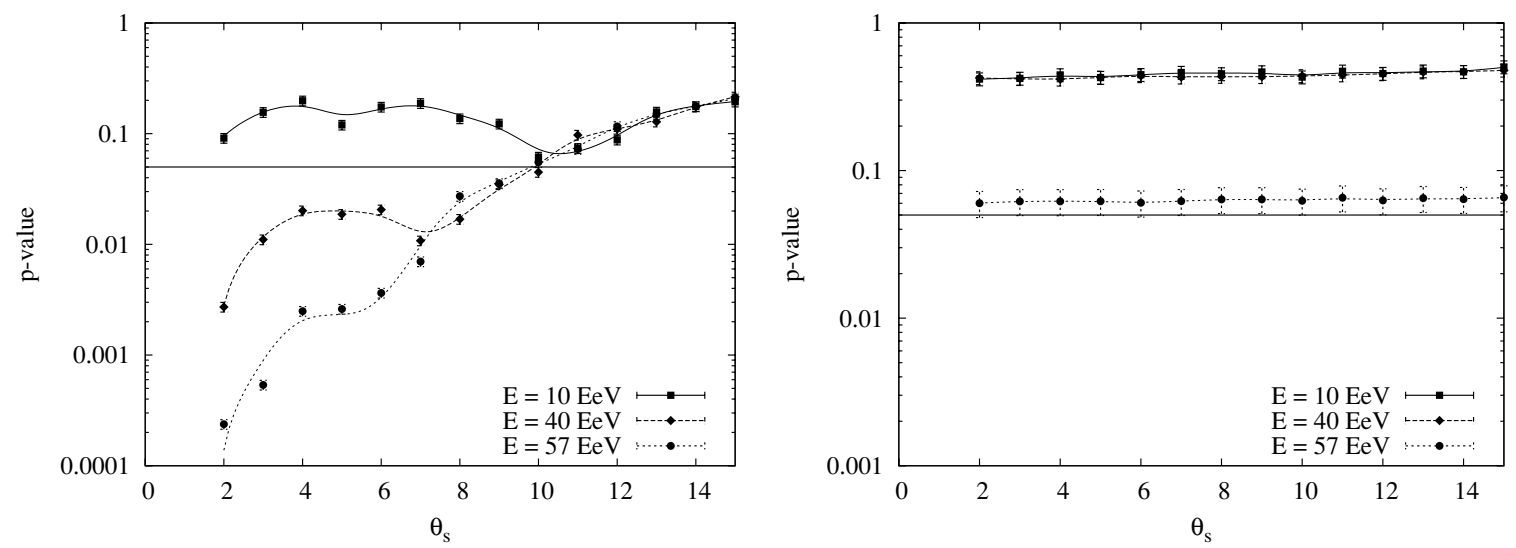

Figure 4. Dependence of $p$-value indicating the level of (in)compatibility between HiRes data and model predictions on the smearing angle $\theta_{\mathrm{s}}$. Solid lines indicate a $p$-value equal to 0.05 , below which the model is ruled out at $95 \% \mathrm{CL}$. The points represent numerical results (with estimated uncertainties of $20 \%$ ); the lines are smooth interpolations between these points. Left panel: data vs. matter tracer model; right panel: data vs. isotropic distribution. 


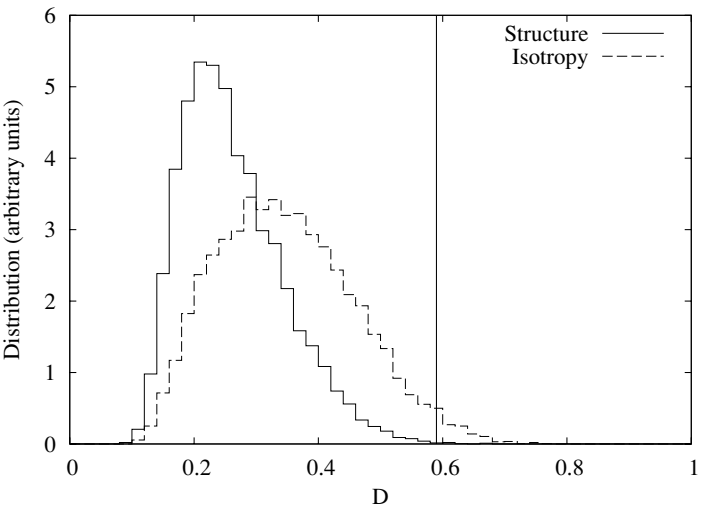

Figure 5. Model distribution of test statistic $D$ when testing the matter tracer model, for both the matter tracer model ("Structure") and an isotropic flux distribution ("Isotropy"). Here $E=57 \mathrm{EeV}$ and $\theta_{\mathrm{s}}=3.2$. The vertical line indicates the observed value $D_{\mathrm{obs}}=0.59$

Since AGNs are tracers of the distribution of matter in the universe, the PAO result is suggestive of a more general correlation between UHECRs and the local structure of the universe on an angular scale of a few degrees. The methods presented in this Letter allow a check on the existence of such correlations in the HiRes data.

The results presented in the previous section disfavor a correlation between UHECRs and the local structure of the universe. In fact, the flux sampling test yields $p$-values smaller than $10^{-2}$ for the matter tracer model with $\theta_{\mathrm{s}} \lesssim 6^{\circ}$, with a $p$-value of $7 \times 10^{-4}$ for $\theta_{\mathrm{s}}=3.2$. (Note that $\theta_{\mathrm{s}}$ is not in 1:1 correspondence with $\psi$; both quantities are however representative of the angular scale of the problem.) Focusing on the case of $\theta_{\mathrm{s}}=3.2$ in more detail, Figure 5 shows the distribution of the test statistic $D$ for the matter tracer model and for an isotropic flux for this smearing angle. The vertical line shows the value $D_{\text {obs }}=0.59$ obtained for the HiRes data. This demonstrates the strong incompatibility between HiRes data and the matter tracer model for smearing angle $\theta_{\mathrm{s}}=3.2$ and threshold energy $E=57 \mathrm{EeV}$.

\section{CONCLUSIONS}

To summarize, we have confronted the stereo data collected by the HiRes experiment with predictions of the matter tracer model, a generic model of cosmic-ray origin and propagation. The model assumes a large number of cosmic-ray sources within $250 \mathrm{Mpc}$ whose distribution traces that of matter, and relatively small deflections characterized by a single parameter, the typical deflection angle $\theta_{s}$. We have found that the HiRes data with energy thresholds $E=40 \mathrm{EeV}$ and $E=57 \mathrm{EeV}$ are incompatible with the matter tracer model for $\theta_{s}<10^{\circ}$ at $95 \% \mathrm{CL}$. With an energy threshold $E=10 \mathrm{EeV}$ the HiRes data are compatible with the matter tracer model. At all three energy thresholds, the data are compatible with an isotropic flux at 95\% CL.

Large deflections of UHECR, as favored by our results, may be due to strong magnetic fields (Galactic or extragalactic), chemical composition different from pure protons assumed in this Letter, or both. However, the composition of cosmic rays at these energies and from these directions has been measured by the HiRes experiment in Abbasi et al. (2009) and is wholly consistent with a light, mostly protonic composition.

In the present analysis, we have treated the deflections as random and Gaussian, which is only appropriate for small deflection angles and a limited number of events. The actual deflections are expected to contain a coherent component due to the Galactic magnetic field. With the accumulation of UHECR events by PAO in the Southern hemisphere and by Telescope Array in the Northern hemisphere, our analysis will become sensitive to the nature of deflections and thus, with proper modifications of the statistical procedure, may give direct access to the parameters of cosmic magnetic fields.

This work is supported by the National Science Foundation under contracts NSF-PHY-9321949, NSF-PHY-9322298, NSF-PHY-9974537, NSF-PHY-0071069, NSF-PHY-0098826, NSF-PHY-0140688, NSF-PHY-0245328, NSF-PHY-0307098, and NSF-PHY-0305516, Department of Energy grant FG0392ER40732, the BSP under IUAP VI/11, the FNRS contract 1.5.335.08, and the IISN contract 4.4509.10. We gratefully acknowledge the contribution from the technical staffs of our home institutions and thank the University of Utah Center for High Performance Computing for their contributions. The cooperation of Colonels E. Fisher, G. Harter, G. Olsen, the US Army, and the Dugway Proving Ground staff is appreciated.

\section{REFERENCES}

Abbasi, R. U., et al. (HiRes) 2005, ApJ, 622, 910

Abbasi, R. U., et al. (HiRes) 2006, ApJ, 636, 680

Abbasi, R. U., et al. (HiRes) 2007, Astropart. Phys., 27, 370

Abbasi, R. U., et al. (HiRes) 2008a, Phys. Rev. Lett., 100, 101101

Abbasi, R. U., et al. 2008b, Astropart. Phys., 30, 175

Abbasi, R. U., et al. 2009, arXiv:0910.4184

Abraham, J., et al. (Pierre Auger) 2007, Science, 318, 938

Abraham, J., et al. (Pierre Auger) 2008a, Phys. Rev. Lett., 101, 061101

Abraham, J., et al. (Pierre Auger) 2008b, Astropart. Phys., 29, 188

Abu-Zayyad, T., et al. (HiRes) 1999, in Proc. 26th International Cosmic Ray Conference, Vol. 5, ed. D. Kieda, M. Salamon, \& B. Dingus (Salt Lake City, UT: IUPAP), 349

Abu-Zayyad, T., et al. 2000, Phys. Rev. Lett., 84, 4276

Abu-Zayyad, T., et al. (HiRes-MIA) 2001, ApJ, 557, 686

Bellido, J. A., Clay, R. W., Dawson, B. R., \& Johnston-Hollitt, M. 2001 , Astropart. Phys., 15, 167

Bergman, D. R., (HiRes) 2007, Nucl. Phys. B, 165, 19

Bird, D. J., et al. (HiRes) 1993, Phys. Rev. Lett., 71, 3401

Bird, D. J., et al. (HiRes) 1999, ApJ, 511, 739

Boyer, J. H., Knapp, B. C., Mannel, E. J., \& Seman, M. 2002, Nucl. Instrum. Methods, A482, 457

Gorbunov, D. S., Tinyakov, P. G., Tkachev, I. I., \& Troitsky, S. V. 2004, JETP Lett., 80,145

Greisen, K. 1966, Phys. Rev. Lett., 16, 748

Hanlon, W. 2008, PhD thesis, Univ. of Utah (http://www.cosmic-ray.org/thesis/ hanlon.html)

Hayashida, N., et al. 1996, Phys. Rev. Lett., 77, 1000

Hayashida, N., et al. (AGASA) 1999, Astropart. Phys., 10, 303

Heck, D., Schatz, G., Thouw, T., Knapp, J., \& Capdevielle, J. N. 1998, Tech. Rep. FZKA 6019

Kalmykov, N. N., Ostapchenko, S. S., \& Pavlov, A. I. 1997, Nucl. Phys. B, 52, 17

Kashti, T., \& Waxman, E. 2008, J. Cosmol. Astropart. Phys., JCAP05(2008)006

Kewley, L. J., Clay, R. W., \& Dawson, B. R. 1996, Astropart. Phys., 5, 69

Koers, H. B. J., \& Tinyakov, P. 2008, Phys. Rev. D, 78, 083009

Koers, H. B. J., \& Tinyakov, P. 2009a, J. Cosmol. Astropart. Phys., JCAP04(2009)003

Koers, H. B. J., \& Tinyakov, P. 2009b, MNRAS, 399, 1005

Santos, E. M., (Pierre Auger) 2007, in Proc. 30th International Cosmic Ray Conference, Vol. 4, ed. R. Caballero, et al. (Mexico City: Universidad Nacional Autónoma de México), 171

Stanev, T., Biermann, P. L., Lloyd-Evans, J., Rachen, J. P., \& Watson, A. A. 1995, Phys. Rev. Lett., 75, 3056

Véron-Cetty, M.-P., \& Véron, P. 2006, A\&A, 455, 773

Zatsepin, G. T., \& Kuzmin, V. A. 1966, JETP Lett., 4, 78 